\title{
微动界面连续干摩擦行为的分子动力学模拟”
}

\author{
潘帅航 ${ }^{1,2}$ 尹 念 ${ }^{1}$ 张执南 1,3 \\ (1. 上海交通大学机械与动力工程学院 上海 200240; \\ 2. 加州大学洛杉矶分校机械与航空航天工程系 洛杉矶 90095 美国; \\ 3. 中国科学研究院兰州化学物理研究所固体润滑国家重点实验室 兰州 730000)
}

\begin{abstract}
摘要: 为探究微动界面连续干摩擦过程中的分子运动规律, 以晶体硅-金刚石耦合微动摩擦副为研究对象, 建立了单凸体固固接触模型, 并以摩擦力响应和摩擦力垂直方向( $\mathrm{z}$ 向)力学响应为表征量进行了分子动力学模拟分析。结果表明, 在多次摩擦 接触间隙, 微动界面之间存在连续、波动的摩擦力学响应; 连续摩擦接触过程中, 受黏滑现象、单凸体变形回复以及界面上 被磨损原子重新分布释放应力等过程影响, 摩擦间隙仍会产生一定的 $z$ 向力学响应, 该力学响应的大小甚至会超过之后摩擦 接触状态的力学响应, 从而影响到固-固耦合微动界面的摩擦力学特性。
\end{abstract}

关键词: 分子动力学; 微动界面; 连续摩擦; 微动磨损

中图分类号: TH133

\section{Molecular Dynamics Simulation for Continuous Dry Friction on Fretting Interfaces}

\author{
PAN Shuaihang ${ }^{1,2}$ YIN Nian $^{1}$ ZHANG Zhinan ${ }^{1,3}$
}

(1. School of Mechanical Engineering, Shanghai Jiao Tong University, Shanghai 200240;

2. School of Mechanical \& Aerospace Engineering, University of California-Los Angeles, Los Angeles, 90095 USA;

3. State Key Laboratory of Solid Lubrication, Lanzhou Institute of Chemical Physics, Chinese Academy of Science, Lanzhou 730000)

\begin{abstract}
Aimed at exploring the continuous dry friction behavior at fretting interface, targeted at crystalline silicon-diamond coupled interface, the fretting interface model with single asperity for dry friction analysis is set up using LAMMPS (i.e., molecular dynamics simulation tool, MD). The micro-motion process is shown with clear images and symbolized with friction force response and the normal force. It is should be noted that during the whole process, including the intervals between direct friction contacts, there exists continuously turbulent force response. The results indicate that during the continuous single asperity contacts, influenced by the factors of stick-slip effects, deformation-recovery process and stress release of wore atoms, the friction contact intervals show friction response, possibly stronger than that of the direct friction contacts to the extent that the frictional characteristics of the coupled solid fretting interfaces can be affected.
\end{abstract}

Key words: molecular dynamics simulation; fretting interfaces; continuous friction; fretting wear

\section{0 前言}

卫星等航天器受发射和在轨工作工况影响, 其 连接件的接触副(连接面)将不可避免地产生难以察 觉的微幅相对运动, 这些微幅相对运动将不可避免

* 国家自然科学基金(51575340)、固体润滑国家重点实验室开放基金 (LSL-1604)和上海航天技术研究院-上海交大航天先进技术联合研究中 心(USCAST2016-13)资助项目。20170218 收到初稿, 20170712 收到修 改稿
地导致微动摩擦学问题, 从而诱发微动损伤甚至失 效, 这会影响到航天器的高精度稳定度、长寿命可 靠性。而此类损伤和失效机理由于观测和试验的难 度较大、高相似的实验条件重复率低且难获得等因 素，目前还缺少有效的研究手段。

分子动力学模拟(Molecular dynamics simulation, $\mathrm{MD}$ )技术的发展, 极大地促进并简化了从原子的角 度对于物质结构、性质和性能的模拟与分析 ${ }^{[1-4]}$ 。例 如, 李俊烨等 ${ }^{[5]}$ 利用分子动力学模拟手段研究了单 
晶铜材料微切削过程, 发现了颗粒对工件做功过程 中的能量转换过程以及颗粒速度和工作能量的关 系。MD 技术的发展使得基于仿真模拟探究物质摩 擦的微观本质成为了可能 ${ }^{[6-8]}$ 。借助 MD 可从微纳尺 度理解微动界面的摩擦学行为机理, 可以为失效预 测及其防护设计提供参考依据; 同时, 由于分子动 力学工具能够更好地实现在各实际工况下对于摩擦 行为影响因素的解耦分析, 因此能够得到微动摩擦 中更为本征的力学响应特性和分析结果。然而, 由 于 MD 仿真工具尚未成熟, 计算量较为庞大, 且仿 真结果和相关参数需要从微观尺度 (量子力学)过渡 到介观、宏观尺度(经典力学)才能对应实际工况, 因此目前 $M D$ 模拟在摩擦学上的运用还存在着一定 的不足, 还需要进一步的探索。

目前, MD 模拟的运用主要集中在图像化地复 现实验结果, 一般仅关注界面上单次摩擦的作用情 况, 未能结合实际情况对多次摩擦和摩擦间隙的状 态进行有效分析, 同时还忽略了个体原子可能的重 要作用 ${ }^{[9-11]}$ 。例如, ZHENG 等 ${ }^{[9-10]}$ 在利用分子动力 学探究单凸体摩擦润滑作用的影响时仅进行了单次 接触的应力、摩擦力分析。LIU 等 ${ }^{[11]}$ 采用 MD 模拟 研究了金属单次刻划过程, 但未能明确指出连续刻 划中摩擦力的变化趋势。摩擦学领域的分子动力学 模拟主要是为了更好地探究微观尺度下的摩擦行为 及影响参数, 一般使用固定压强来设定仿真边界条 件。例如, SHA 等 ${ }^{[12]}$ 和 DAI 等 ${ }^{[13]}$ 在研究 DLC 界面 之间的摩擦作用时, 始终把摩擦力的法向力学响应 作为可控参量来进行设定。VARGONEN 等 ${ }^{[14]}$ 在探 究微磨损的作用机理时施加固定的法向压力, 以此 实现摩擦过程中摩擦法向的运动。实际上, 针对不 同工况的仿真边界条件的确定仍有待商榷。

在干摩擦条件下, 微动界面的实际接触发生在 离散的单凸体上，当单凸体相互接近时，原子间的 作用力使单凸体产生黏着; 当发生相对滑动时, 黏 着力和其他作用力共同构成了摩擦力。因此, 针对 单凸体的摩擦研究是探究微动界面摩擦现象的基 础。本文针对微动界面单凸体连续干摩擦行为开展 分子动力学建模与分析, 为探索精密摩擦副微动磨 损的退化机理和本质, 以及预测精密摩擦副寿命和 微动损伤防护提供基础。

\section{1 分子动力学建模}

\section{1 分子动力学模型}

考虑到作为单质形式存在的金刚石和晶体硅, 虽然在航天器部件减摩和精仪制造运用上已经得到
了足够的重视, 但是随着微观研究的深入, 其在 摩擦学领域的力学响应等问题并没有从微观角度 得到明确的解释, 也因此在该领域难以做到从微 观到宏观机理的跨接, 因此, 在本文的分子动力 学仿真研究中, 以金刚石和晶体硅为摩擦配副材 料并构造单凸体结构进行分子动力学建模, 所建 模型如图 1 所示。

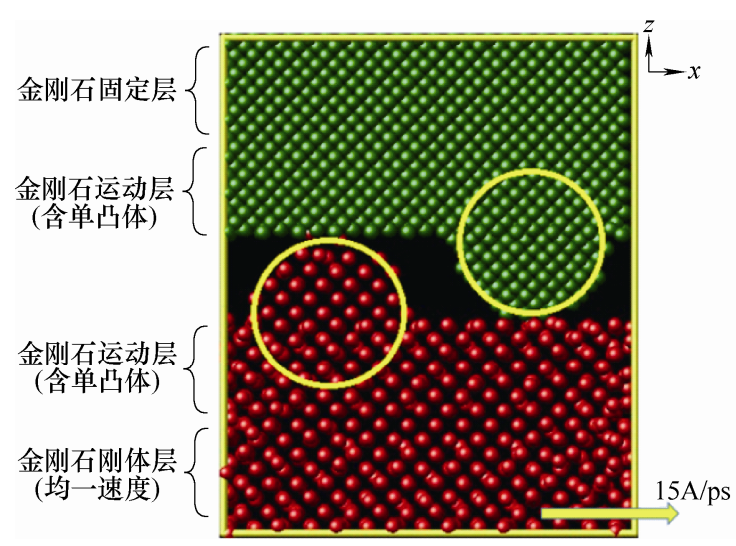

图 1 微动界面单凸体分子动力学模型

图 1 中，仿真区域尺寸为 $40 \AA \times 40 \AA \times 48 \AA$, 模型中上半部分为金刚石原子, 下半部分为晶体硅 原子，其中金刚石的莫氏硬度为 10.0 , 晶体硅的莫 氏硬度为 7.0, 体积模量 $E_{v}=100 \mathrm{GPa}$, 泊松比 $v=0.22$ 。 由于在原子尺度进行模拟, 故将单凸体模型简化为 半球形。在进行摩擦运动仿真时, 模型从上到下分 为四层, 分别为金刚石刚体层(即固定层), 金刚石 运动层(含单凸体), 硅运动层(含单凸体)和硅刚体层 (即均一速度运动); 运动速度设定为 $15.0 \AA / \mathrm{ps}$, 以 实现在仿真时间内的多次摩擦; 单凸体均简化为半 径为 $8 \AA$ 的半球形, 此外为了更好地适应原子运动 和整体的运动学仿真, 考虑到晶体硅的晶格常数为 $5.43 \AA$, 设定原子的截断半径为 $3.7 \AA$ 。应当注意到, 单凸体虽然简化为半球形, 实际模拟中, 因为每个 原子占有一定的体积和空间, 所以各单凸体均按阶 梯状分布、且在能量最小化后因为相互作用的设定 而存在碳一硅的成键, 更接近真实的接触工况。

\section{2 势场设定}

为了反映分子间作用力的影响, 两接触界面之 间使用 Lennard-Jones 势场 ${ }^{[15-17]}$; 金刚石和晶体硅均 使用 Tersoff 势场 ${ }^{[17-19]}$ 。Tersoff 势场势能的描述方程 如公式(1) (8)所示。

$$
E=\frac{1}{2} \sum_{i} \sum_{j \neq i} V_{i j}
$$

$$
\begin{gathered}
V_{i j}=f_{c}\left(r_{i j}\right)\left[f_{r}\left(r_{i j}\right)+f_{a}\left(r_{i j}\right) \times b_{i j}\right] \\
f_{r}(r)=A \exp \left(-\lambda_{1} r\right)
\end{gathered}
$$




$$
\begin{gathered}
f_{a}(r)=-\operatorname{Bexp}\left(-\lambda_{2} r\right) \\
f_{c}(r)=\left\{\begin{array}{cc}
1 & r<R-D \\
\frac{1}{2}-\frac{1}{2} \sin \left(\frac{\pi}{2} \frac{r-R}{D}\right) \quad R-D \leqslant r<R+D \\
0 \quad r \geqslant R+D
\end{array}\right. \\
b_{i j}=\left(1+\beta^{n} \zeta_{i j}^{n}\right)^{-1 / 2 n} \\
g(\theta)=\sum_{k \neq i, j} f_{c}\left(r_{i j}\right) g\left(\theta_{i j k}\right) \exp \left[\lambda_{3}^{m}\left(r_{i j}-r_{i k}\right)^{m}\right] \\
\left(1+\frac{c^{2}}{d^{2}}-\frac{c^{2}}{\left[d^{2}+\left(\cos \theta-\cos \theta_{0}\right)^{2}\right.}\right)
\end{gathered}
$$

式中, $r$ 为原子之间的相对距离; $f_{r}$ 为原子间排斥 力作用; $f_{a}$ 为原子之间吸引力作用; $f_{c}$ 为截断作用 系数, 表示距离对相互作用的影响; $D$ 为晶格常数, $R$ 是反映原子相互作用范围的基本参数 $(R=0.5 D) ; \theta$ 为键角, $\theta_{0}$ 为能量最小状态的键角; 其余参数 $A$, $B, \lambda_{1}, \lambda_{2}, \beta, \zeta, n, m, c, d$ 和三体作用参数 $\gamma$ 为与设定物质对应的常参数。

\section{2 结果与讨论}

\section{1 连续干摩擦行为分析}

基于图 1 所建立的分子动力学模型, 以 LAMMPS 为工具编写分子动力学模拟程序并实施 多次摩擦接触的分子动力学模拟。为了保证仿真模 拟中摩擦现象的可重复性, 在 $0 \sim 30 \mathrm{ps}$ 的时间范围 内, 进行了多次摩擦动力学模拟; 考虑到实际摩擦 工况, 在对摩擦间隙力学响应进行讨论时, 重点关 注 $0 \sim 10 \mathrm{ps}$ 的摩擦力学现象。如图 2 所示, 在设定 的仿真时间内共实现有效、明显的摩擦接触行为共 计 3 次。其中图 $2 \mathrm{a}$ 为摩擦接触开始, 图 $2 \mathrm{~b}$ 为第一 次摩擦变形, 图 2c 第二次摩擦接触, 图 2d 为第三 次摩擦接触。

如图 2a 所示, 由于金刚石和晶体硅之间的宏观 硬度差异较大, 在第一次摩擦接触时, 金刚石单凸 体虽有微小变形, 但整体未发生明显磨损; 晶体硅 则同时发生了黏附、挤压变形和脱附三种作用, 分 别对应摩擦中的变形、磨损和应力释放, 其中脱附 的发生是因为晶体硅与碳成键断裂, 并再次随晶体 硅运动层运动。单凸体第二次和第三次摩擦接触重 复上述过程。

由图 2b 和 2c 可以看出, 第一次摩擦过后, 晶 体硅的单凸体磨损严重, 高度从 $8 \AA$ 减少到 3-4 而部分硅原子则黏附在金刚石单凸体表面, 连续摩 擦过程中金刚石单凸体的接触面积没有明显增大。
从图 2 中还可以看出, 金刚石单凸体表现为整体 性微弯曲, 其中以图 2d 中金刚石单凸体微弯曲最 为明显。

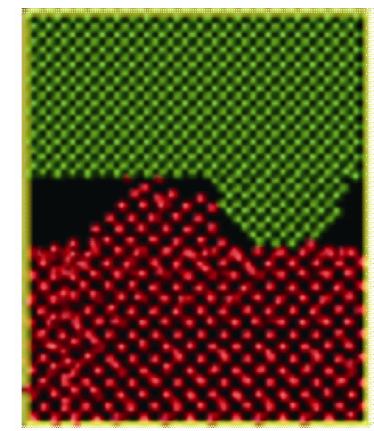

(a) 摩擦接触开始

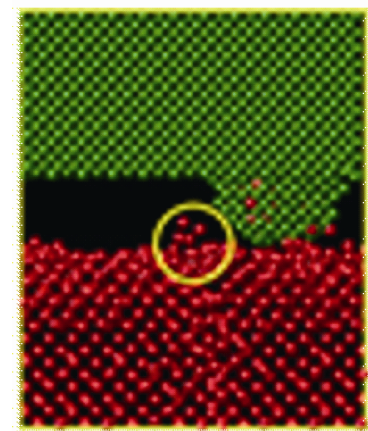

(c) 第二次摩擦接触

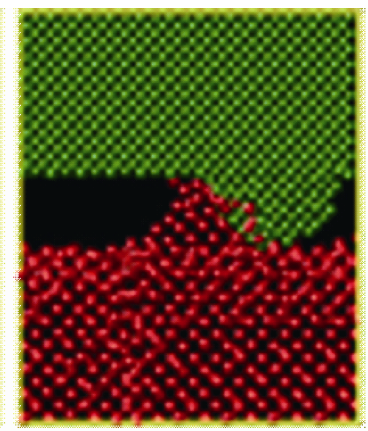

(b) 第一次摩擦变形

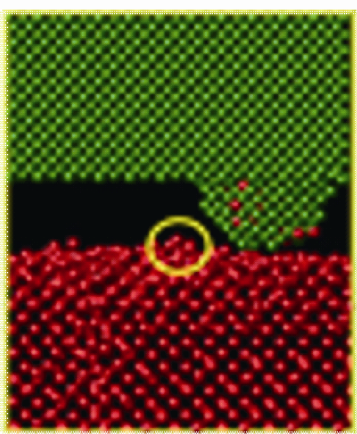

(d) 第三次摩擦接触
图 2 多次摩擦接触的分子动力学模拟

未能脱附的硅原子与金刚石原子一起参与到对 硅单凸体其余部分的磨损, 而随着微动摩擦的多次 发生、晶体硅的单凸体逐渐消耗, 这部分硅不再脱 附; 第二次到第三次摩擦发生时重复上述过程, 上 述作用过程如图 3 所示。

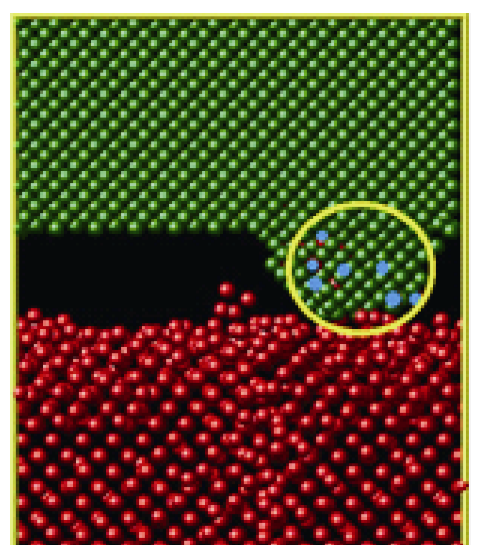

图 3 第一次到第二次摩擦接触间隙未脱附硅原子分布区域

此外, 图 3 显示, 在摩擦接触过程(含摩擦接触 间隙)中，虽然单凸体消耗减小了摩擦磨损带来的力 学响应, 但除了硅原子的黏附现象外, 晶体硅的晶 格还出现了明显的挤压变形 (表现为在除此摩擦接 触开始后晶格排列的无序性)。

这主要是因为在摩擦过程中, 随着金刚石和晶 
体硅的靠近、摩擦接触, 两者受到挤压时的能量通 过(弹性)形变储存, 由于金刚石较大的弹性模量, 金刚石的晶格变形会小于晶体硅; 再者, 由于硬度 差异(宏观硬度和微观硬度), 且金刚石的晶格常数 小于晶体硅, 金刚石会倾向于通过进一步挤压晶体 硅、恢复形变释放部分能量。上述微观影响因素共 同导致了晶体硅明显的晶格变形。

该微观影响因素也是摩擦接触间隙出现摩擦响 应的主要因素, 将在第 2.2 节中结合力学响应响应 的数据分析进行进一步说明。

\section{2 摩擦力学响应分析}

图 4 给出了 $\mathrm{z}$ 向力学响应的采集方式, 由于 考虑间距固定的接触工况, 特设定 $z$ 向保持高度 不变, 在单凸体接触界面, 通过 $z$ 向相对力学响 应获得摩擦力法向力响应。图 3 中取稳定时刻 $z$ 向力学响应为 0 , 并在相对运动开始后开始记录 $\mathrm{Z}$ 向相对力学响应。根据仿真区域尺寸 $40 \AA \times 40 \AA$ $\times 48 \AA$ 以及模型相对运动速度 $15 \AA / p s$, 并结合摩 擦力波动周期, 可以对晶体硅的晶格常数进行粗 略估算。

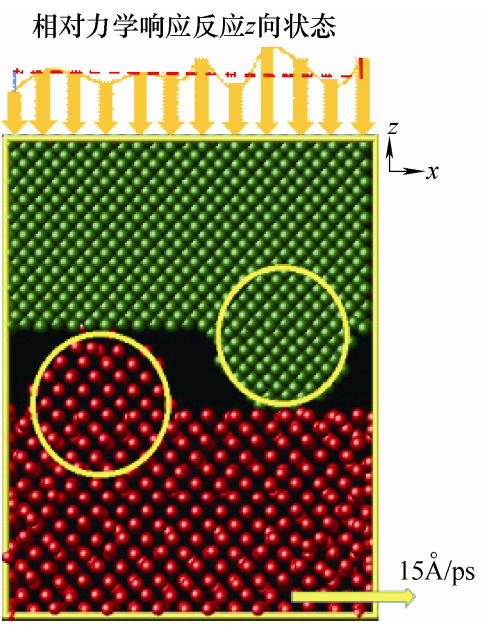

图 $4 z$ 向力学响应采集方式

考虑多次摩擦, 且存在未脱附硅原子和其余硅 原子之间的挤压、摩擦作用, 对界面摩擦力和界面 $\mathrm{z}$ 向相互作用力进行了分析, 其结果如图 5 所示。 其中图 5a 为金刚石-晶体硅界面之间的摩擦力学响 应, 图 5b 为金刚石-晶体硅界面 $z$ 向相互作用力, 图中虚线表示摩擦接触间隙状态。

由图 5 可以看出, 金刚石-晶体硅耦合界面在摩 擦过程中的界面力学响应相对较小。在每次摩擦接 触过程中, 摩擦力均表现出规律性循环波动, 且摩 擦力的波动峰值出现的频率高于 $z$ 向力学响应的幅 值波动频率; 根据图 3 的 $\mathrm{z}$ 向相对力学响应曲线的 峰值距离, 结合运动速度为 $15.0 \AA / \mathrm{ps}$, 计算得摩擦 力幅值波动的周期为 $0.3 \sim 0.4 \mathrm{ps}$, 由此计算得到的 晶体硅晶格常数为 $4.5 \sim 6.0 \AA$, 在动态仿真误差范 围内较好地符合了晶体硅试验测定的晶格常数 5.43 $\AA$, 与界面摩擦存在黏滑现象的假设相吻合, 验证 了模型设定和运动仿真的正确性。由于 MD 模拟中 保持了高度不变, 每次摩擦接触均会产生摩擦力响 应和明显增大的界面相互作用应力, 且随着多次摩 擦的进行, 由于硅单凸体的磨损消耗, 每次摩擦引 起的摩擦力学响应减小。

如 5b 所示, 在第一次摩擦结束到第二次摩擦接 触发生之间, 摩擦界面间的相互作用力仍出现峰值
分布并在 $z$ 向表现为挤压作用, 其压力幅值为 15 $\mathrm{nN}$, 超过了第一次摩擦过程, 结合盒体的面积 $S=40 \AA \times 40 \AA$, 可知接触压强接近 $1 \mathrm{GPa}$ 。这主要 是因为在第一次摩擦过后较多黏附的硅原子突出并 作用在其原界面, 而部分脱附的硅原子在其原界面 进行分散分布、进一步释放应力导致的。随着多次 摩擦的进行, 在第二次摩擦接触结束到第三次摩擦 接触发生前, 由于黏附的硅原子减少, 这一现象已 经相对减弱, 并且摩擦间隙的 $z$ 向力学响应幅值难 以超过第一次摩擦过程中的 $z$ 向力学响应幅值。

此外, 从图 5 中还可以看出, 从第一次摩擦开始 到第三次摩擦接触结束, 摩擦力学响应的峰值均出现 在相应摩擦接触时间段的中后期, 这可以认为是体现 刚体性质的上层金刚石单凸体(含黏附硅原子)在摩擦 接触后期由微变形状态进行变形回复所导致的, 这种 变形回复带来的波动也会对随后的摩擦间隙产生影 响, 导致接触界面的相互作用和较强的力学响应, 尤 其在小分子量模拟时这一影响(即任何一部分原子可 能产生的影响)更加突出。总体而言, 第一次摩擦接触 时单凸体作用较大, 但从第二次摩擦接触开始, 由于 下层单凸体的不断磨损, 故随着每次摩擦进行, 上层 单凸体的弯曲变形不断减小, 因此由其变形回复带来 的对界面相互作用峰值响应的影响也不断削弱。 


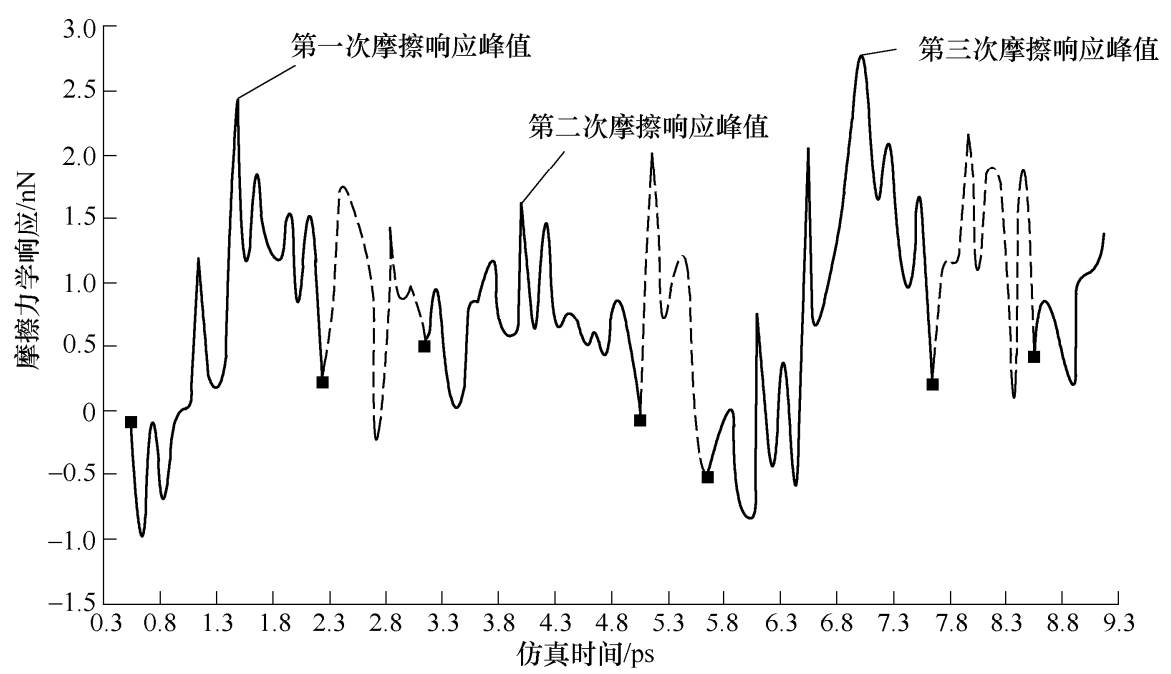

(a) 金刚石一晶体硅界面之间摩擦力

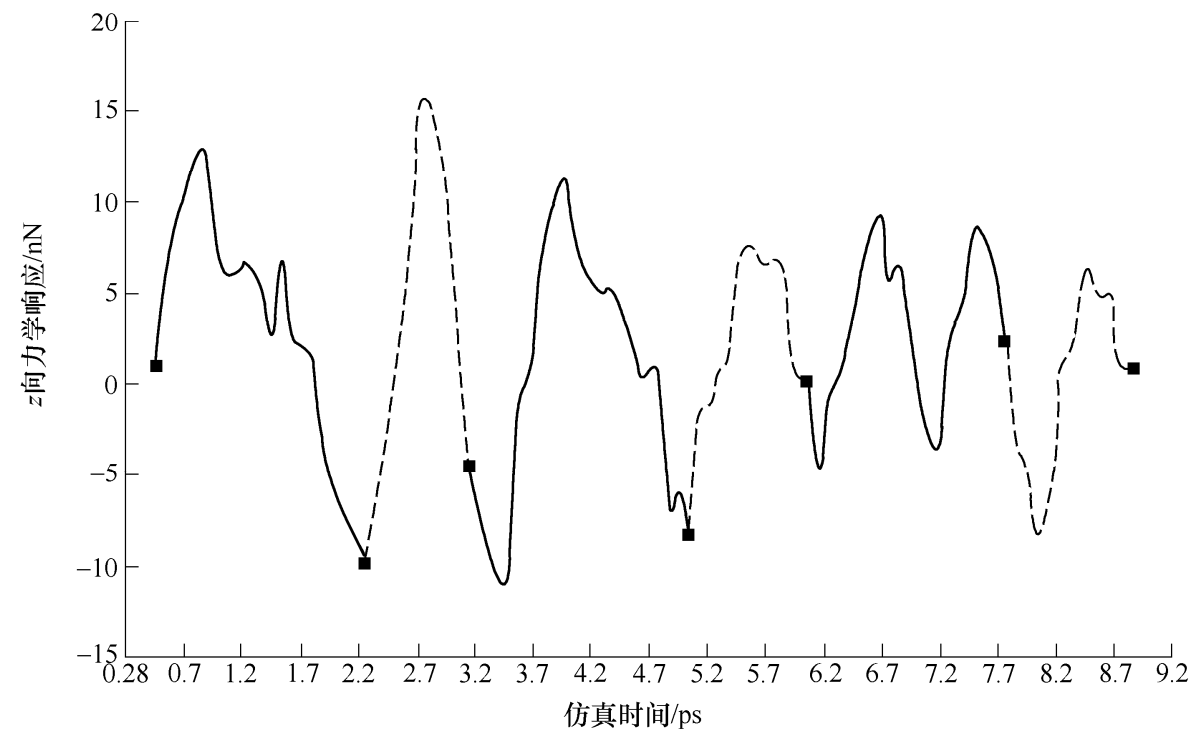

(b) 金刚石一晶体硅界面 $z$ 向相互作用力

图 5 金刚石-晶体硅连续摩擦过程力学曲线(虚线为摩擦接触间隙状态)

\section{3 结论}

(1) 针对晶体硅-金刚石摩擦副的微动摩擦现 象，由于金刚石和晶体硅摩擦接触，两者受到挤压 时的能量通过(弹性)形变储存, 由于金刚石较大的 弹性模量和宏观、微观硬度, 加之金刚石较小的晶 格常数，金刚石会倾向于通过进一步挤压晶体硅、 恢复形变释放部分能量, 导致晶体硅较大的单凸体 磨损和较为明显的晶格变形。上述分析表明, 摩擦 间隙的力学响应出现主要依赖于晶体硅的形变能量 释放和晶格变形的缓慢消除。

（2）总体来说, 针对三维结构固-固界面的微动 摩擦分析需要关注摩擦接触间隙的力学响应。这是 因为在摩擦间隙仍会出现较大的摩擦力和摩擦力法 向力学响应。可以认为这是由于在摩擦接触过程中
粘附后脱附的单凸体原子在界面重新排布、释放摩 擦变形的应力所导致的。

(3) 针对单凸体的摩擦磨损分析, 若摩擦副界 面宏观硬度差异较大，则硬度较大的单凸体发生整 体性的刚性变形和微弯曲, 不会出现单凸体和基体 接触面积增大的情况, 且由于存在微弯曲的变形回 复过程, 会造成摩擦间隙力学响应的较大波动。

\section{参 考 文 献}

[1] 罗旋, 贾维栋, 李超, 等. 材料科学中的分子动力学模 拟研究进展 [J]. 材料科学与工艺, 1993, 4(1): 125-128. LUO Xuan, FEI Weidong, LI Chao, et al. Advance of molecular dynamics simulation in materials science[J]. Material Science and Technology, 1993， 4(1): 125-128.

[2] 王慧, 胡元中, 周鲲, 等. 纳米摩擦学的分子动力学模 拟研究[J]. 中国科学(A 辑), 2001, 31(3): 261-266.

WANG Hui, HU Yuanzhong, ZHOU Kun, et al. 
Molecular dynamics simulation for nanotribology[J]. Science in China (Series A), 2001, 31(3): 261-266.

[3] 㚞康旗, 贾建援. 经典分子动力学模拟的主要技术 $[\mathrm{J}]$. 微纳电子技术, 2005(3): 133-137.

FAN Kangqi, JIA Jianyuan. An overview on classical molecular dynamics[J]. Micronanoelectric Technology, 2005(3): 133-137.

[4] 文玉华, 朱如曾, 周复信, 等. 分子动力学模拟的主要 技术 $[\mathrm{J}]$. 力学进展, 2003, 33(1): 65-73.

WEN Yuhua, ZHU Ruzeng, ZHOU Fuxin, et al. An overview on molecular dynamics Simulation [J]. Advances in Mechanics, 2003， 33(1): 65-73.

[5] 李俊烨, 董坤, 王兴华, 等. 颗粒微切削表面创成的分 子动力学仿真研究 [J]. 机械工程学报, 2016, 52(17): 94-104.

LI Junye, DONG Kun, WANG Xinghua, et al. Molecular dynamics simulation research into generative mechanism of particles micro-cutting surface[J]. Journal of Mechanical Engineering, 2016， 52(17): 94-104

[6] WU Chengda. Molecular dynamics simulation of nanotribology properties of $\mathrm{CuZr}$ metallic glasses[J]. Applied Physics A-Materials Science \& Processing, 2016, 122: 486.

[7] EDER S J , CIHAK-BAYR U, BIANCHI D. Single-asperity contributions to multi-asperity wear simulated with molecular dynamics[J]. Materials Science and Engineering. 2016, 119: p012009.

[8] ARISTIZABAL H D, PARRA P A, LOPEZ P, et al. Atomic-scale simulations of material behaviors and tribology properties for BCC metal film[J]. Chinese Physics B, 2016, 25(1): 010204.

[9] ZHENG X, ZHU H T, KOSASIH B, et al. A molecular dynamics simulation of boundary lubrication: The effect of n-alkanes chain length and normal load[J]. Wear, 2013, 301: 62-69.

[10] ZHENG X, ZHU H T, KIET T A, et al. Roughness and lubricant effect on 3D atomic asperity contact [J]. Tribology Letters, 2014, 53: 215-223.
[11] LIU X M, LIU Z L, WEI Y G. Nanoscale friction behavior of the Ni-film/substrate system under scratching using MD simulation[J]. Tribology Letters, 2012, 46: 167-178.

[12] SHA Z D, SORKIN V, BRANICIO P S, et al. Large-scale molecular dynamics simulations of wear in diamond-like carbon at the nanoscale[J]. Applied Physics Letters, 2013, 103: 073118.

[13] DAI L, SORKIN V, SHA ZD, et al. Molecular dynamics simulations on the frictional behavior of a perfluoropolyether film sandwiched between diamond-like-carbon coatings[J]. Langmuir, 2014，30: 1573-1579.

[14] VARGONEN M, YANG Y J, HUANG L P, et al. Molecular simulation of tip wear in a single asperity sliding contact[J]. Wear, 2013, 307: 150-154.

[15] FENNELL C J, GEZELTER J D. Is the Ewald summation still necessary? Pairwise alternatives to the accepted standard for long-range electrostatics[J]. Journal of Chemical Physics, 2006, 124: 234104.

[16] TERSOFF J. Empirical interatomic potential for carbon, with applications to amorphous carbon[J]. Physical Review Letters, 1988, 61: 2879-2882.

[17] ERHART P, ALBE K. Analytical potential for atomistic simulations of silicon, carbon and silicon carbon[J]. Physical Review B, 2005, 71: 035211.

[18] WANG ZG, ZHENG P, CHEN JX, BAI QS, LIANG YC. Effect of C-C bond breakage on diamond tool wear in nanometric cutting of silicon[J]. Acta Physica Sinica, 2015, 64(19): 198104.

[19] XIONG Q L, TIAN X G. Atomistic simulations of interfacial mechanical characteristics of carbon nanotube/silicon nano composites[J]. Molecular Simulation, 2015, 41(13): 1051-1059.

作者简介: 潘帅航, 男, 1994 年出生。主要研究方向为摩擦-微振动 耦合。

E-mail: luckypsh@g.ucla.edu

张执南(通信作者), 男, 1978 年出生, 博士, 副教授。主要研究方向为 摩擦学设计、设计理论与方法。

E-mail: zhinanz@sjtu.edu.cn 